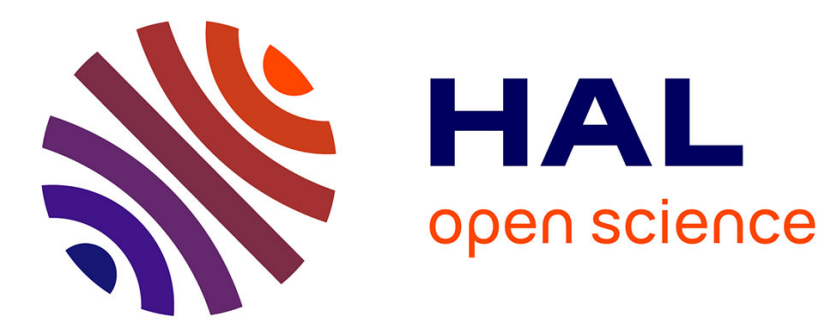

\title{
Introduction. Pour une approche pluridisciplinaire du rituel
}

Sylvie Donnat

\section{To cite this version:}

Sylvie Donnat. Introduction. Pour une approche pluridisciplinaire du rituel. Archimède: archéologie et histoire ancienne, 2014, 1, pp.1-6. halshs-01585279

\section{HAL Id: halshs-01585279 \\ https://shs.hal.science/halshs-01585279}

Submitted on 11 Sep 2017

HAL is a multi-disciplinary open access archive for the deposit and dissemination of scientific research documents, whether they are published or not. The documents may come from teaching and research institutions in France or abroad, or from public or private research centers.
L'archive ouverte pluridisciplinaire HAL, est destinée au dépôt et à la diffusion de documents scientifiques de niveau recherche, publiés ou non, émanant des établissements d'enseignement et de recherche français ou étrangers, des laboratoires publics ou privés. 


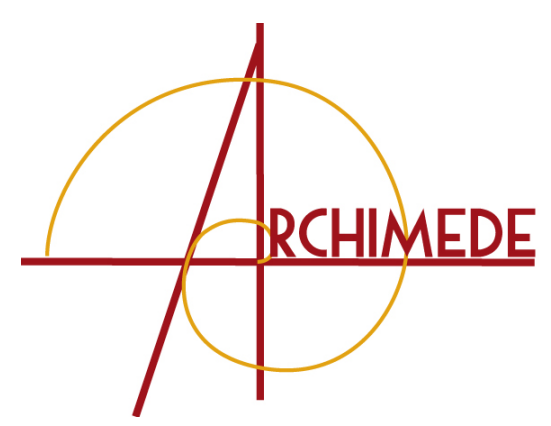

\title{
DOSSIER THÉMATIQUE ARCHÉOLOGIE DU RITUEL
}

\author{
dir. Sylvie Donnat, Philippe Quenet, Catherine Vanderheyde
}

\author{
INTRODUCTION. \\ POUR UNE APPROCHE PLURIDISCIPLINAIRE DU RITUEL
}

\section{RÉSUMÉ}

\author{
Sylvie DONNAT \\ Université de Strasbourg, \\ UMR 7044 Archimède \\ Maître de conférences en égyptologie \\ (Université de Strasbourg), \\ chercheuse associée à l'UMR 8210, \\ ANHIMA, Paris \\ sylvie.donnat@misha.fr
}

Pour tenter de comprendre et de décrire les rites antiques, l'archéologie apparaît comme une des disciplines les mieux à même d'apporter des éléments qui pallieraient, en partie, les silences des sources anciennes. Les avancées en matière de fouille fine et de traitement de données de terrain permettent ainsi de repérer les traces les plus ténues de gestes rituels. En parallèle, les avancées théoriques et méthodologiques dans l'analyse des sources textuelles et iconographiques de natures diverses permettent d'aborder cette documentation avec un regard neuf. Le dialogue, régulièrement renouvelé, des sciences historiques avec l'anthropologie sociale est également un moment important de la recherche, notamment pour la formulation des questions qui sont posées aux sources anciennes.

Mots-CLÉS

Épistémologie, archéologie, anthropologie, histoire,

heuristique, pluridisciplinarité, rituel.
L'étude de l'objet « rituel », en raison de la diversité des traces qu'il est susceptible de laisser, requiert par conséquent une approche pluridisciplinaire.
Describing and understanding an ancient ritual process is a difficult task for the historian. Archaeology is particularly able to identify tenuous evidences of ritual acts, not fully described in ancient texts. New approaches of textual and iconographical sources bring also new evidences. Dialogue among historical sciences and social and cultural anthropology is also important for the raising of relevant scientific issues. "Ritual" is documented by a variety of evidences, and so need a multidisciplinary approach. 
Je remercie Philippe Quenet, Catherine Vanderheyde, Sandra Boehringer et Jean-Marie Husser pour leurs lecture et remarques sur ce texte.

L'historien des religions et des pratiques antiques mesure régulièrement toute la difficulté qu'il y a à se penser comme un ethnologue des civilisations passées en l'absence de toute possibilité d'observation directe des rites. L'archéologie apparaît ainsi comme une voie possible pour apporter des éléments qui pallieraient, en partie, le silence des sources textuelles anciennes et qui permettrait à I'historien, à défaut d'assister à une cérémonie donnée, d'en observer les traces. Dans le milieu francophone, le champ de recherche de l'archéologie du rite a été marqué par l'article de I'historien de la Rome antique John Scheid, «Pour une archéologie du rite », paru en 2000 dans la revue Annales. Histoire, Sciences sociales [1]. L'auteur y faisait le constat que les sources littéraires - sources de prédilection de I'historienétaient suffisantes pour nous dire l'importance du rite dans les religions antiques, mais pas assez précises pour en donner le détail des gestes. Prenant acte des résultats de I'archéologie de I'Europe celtique depuis les années 1980, John Scheid appelait en conséquence de ses vœux le développement d'une archéologie du rite, qui mettrait en œuvre les dernières avancées en matière de fouille fine, d'enregistrement des données et qui ferait largement appel aux sciences dites auxiliaires que sont, entre autres, I'anthropologie physique, l'archéozoologie ou I'archéobotanique. L'archéologie du rite se présente ainsi comme un ensemble de questions posées au terrain, un programme d'investigation archéologique nécessitant I'élaboration de stratégies à même de repérer les traces les plus ténues de l'exécution de gestes rituels [2]. À la suite de l'appel programmatique de John Scheid, plusieurs importantes contributions à l'archéologie du rite

\section{[1] SCHEID 2000.}

[2] Voir la méthodologie exposée dans LePETZ \& VAN ANDRINGA 2008b ; VAN ANDRINGa \& LePETZ 2005, p. 339-340.

[3] Lepetz \& Van Andringa (éd.), 2008a; Scheid (éd.) 2008. Voir encore récemment le volume QuANTIN (éd.) 2012 sur «l'archéologie des religions antiques ».

[4] Voir en particulier le programme des EFA-EFR 2012-2016 sur I'archéologie du culte dans les sanctuaires méditerranéens, dirigé par W. Van Andringa et S. Huber : http://www.ecole-francaise.it/fr/q/d/ Programme_2.5.2.pdf (consultation juin 2014).

[5] KYRIARDIS 2005 ; InSOLL (éd.) 2011 ; WilbuRn 2012 (« Archaeology of magic »).

[6] Mylonopoulos, Roeder (éd.) 2006, paru dans le cadre du programme SFB 619 «Ritual Dynamik» (2002-2013 : http:// www.ritualdynamik.de - consultation juillet 2014 -, avec liste des publications du programme).

[7] Voir encore le volume collectif Approaching Rituals in Ancient Cultures, Ambos \& Verderame (éd.) 2013.

[8] Pour un historique de ce champ de la recherche en sciences humaines, voir les références citées dans MyLonopoulos \& RoEder 2006, p. 9, n. 2. Voir aussi les remarques dans Ambos \& Verderame sont parues [3] et des programmes sont actuellement en cours [4]. La production de ce domaine de recherche ne se limite bien évidemment pas à la recherche francophone, comme l'atteste la littérature récente abondante sur ce sujet, notamment en langue anglaise [5] ou en langue allemande [6]. Toutes ces contributions [7] s'inscrivent, de manière plus large, dans le productif champ des sciences humaines (commun à l'anthropologie, la sociologie, la psychologie et l'histoire des religions) des études sur le rite (ritual studies) [8]. Si l'essor de l'archéologie du rite doit beaucoup aux avancées des techniques de l'archéologie et de ses sciences auxiliaires, le dialogue régulièrement renouvelé entre les sciences historiques et les sciences sociales a en effet joué et joue un rôle déterminant. Pour rester dans le domaine francophone, la démarche anthropologique des chercheurs autour des hellénistes Jean-Pierre Vernant et Marcel Detienne, par exemple, a eu un fort impact [9], et l'expérience collective comparatiste entre historiens et ethnologues, qu'ils ont initiée, se poursuit [10]. L'archéologie préhistorique, qui a dû élaborer une approche spécifiquement matérielle des pratiques religieuses, a pour sa part une histoire propre de ses liens avec l'ethnologie, à travers la figure d'André Leroi-Gourhan [11] et I'ancrage académique de la discipline.

C'est une rencontre scientifique qui est à l'origine du présent dossier: les deux journées du séminaire interdisciplinaire organisé par le département d'Archéologie de I'université de Strasbourg-UMR 7044 sur I'« Archéologie du rituel » en 2013 [12]. L'objectif des contributeurs est de proposer une réflexion sur tous les types de vestiges matériels que peut laisser une pratique humaine qui

2013, p. 11 sur les liens avec I'histoire.

[9] Par exemple dans le cadre des travaux sur le sacrifice antique : Detienne \& Vernant 1979. Cf. la contribution d'Anne Jacquemin dans ce dossier. Voir aussi les remarques de J. Scheid dans le prologue à I'ouvrage collectif Pour une archéologie du rite (SCHEID 2008, p. 5) sur l'importance des enseignements de J.-P. Vernant et G. Dumézil à propos des rites antiques.

[10] Sur l'actualité et la postérité de la démarche comparatiste initiée par J.-P. Vernant et M. Detienne, voir CARASTRO, Guenzi \& ILDEFONSE 2012.

[11] LeROI-Gourhan 1964, en particulier p. 73-76 et p. 136-141, pour la question de la connaissance des rites préhistoriques, avec une discussion critique sur le recours à un « comparatisme sommaire » avec les faits ethnographiques (ibid., p. 4). Cf. TESTART 2006 pour une réflexion sur la collaboration entre archéologie (préhistorique) et anthropologie sociale.

[12] Le choix du thème du Séminaire interdisciplinaire d'Archéologie 2013 a été fait pour adosser ces deux journées au thème transversal II de I'UMR 7044 «Gestes rituels: traces matérielles et interprétations » (http://archimede.unistra.fr/programmes-derecherche/programmes-transversaux/les-gestes-rituels-tracesmaterielles-et-interpretations/) 
relève pour une large part de «l'immatériel », puisque constituée, en grande partie, de gestes exécutés et de paroles prononcées [13]. Le terme « rituel » est utilisé ici pour suggérer que la perspective est celle de l'approche matérielle de tout « ce qui a trait au rite » : les espaces, les officiants, les discours, les images, les objets, etc. Le « rite », en tant que catégorie scientifique, a pour sa part été défini comme désignant un type d'action singulière, régulée selon des modalités spécifiques [14], et constituant pour les participants une expérience de référence, hautement évocatrice [15]. Qu'il soit religieux ou non [16], le rite a la caractéristique d'être composé en grande partie d'éléments intangibles : des gestes, des paroles, des sons, des odeurs, des relations... Ces actions impliquent néanmoins l'existence d'un espace spécifique où elles puissent prendre place, de participants et d'objets à manipuler. La question qui se pose donc à l'archéologue, et à l'historien, est celle des témoignages matériels que peuvent laisser les différents aspects du rite :

- sa dimension spatiale (les lieux, les structures, les espaces de circulation...),

- sa dimension temporelle (indices matériels du moment de l'exécution, de la succession des épisodes rituels...),

- la question de ses agents (humains, animaux, ou entités de l'invisible...),

- celle des ustensiles utilisés et des objets manipulés,

- celle des gestes opérés et de leurs résidus éventuels (restes humains et fauniques, résidus carbonisés, objets brisés, cendres, patine sur un objet, etc.).

Même si l'approche privilégiée est celle de l'archéologue, il est important que la question des textes et des images soit considérée avec attention, puisque ces deux types d'objets sont susceptibles tout autant de proposer un discours extérieur sur le rite que de constituer des éléments de la procédure rituelle, par leur présence dans l'espace cérémoniel (inscriptions épigraphiques, programme décoratif...), par l'assistance qu'ils peuvent apporter aux officiants lors du déroulement du rite (manuel sacerdotal...), par leur capacité à construire la présence d'un agent (images rituelles), etc.

[13] Levi-Strauss 1971, p. 600 : «Comment définira-t-on alors le rituel ? On dira qu'il consiste en paroles proférées, gestes accomplis, objets manipulés indépendamment de toute glose ou exégèse (...) on posera trois questions dont toute l'interprétation théorique du rituel dépend : au cours des rites, de quelle manière distinctive parle-t-on ? Comment gesticule-t-on ? Et quels critères particuliers président au choix des objets rituels et à leur manipulation ? » (Je remercie Salomé Deboos, MCF Anthropologie sociale, Strasbourg, pour avoir attiré mon attention sur cette citation ; lire aussi plus généralement, l'ensemble du paragraphe, ibid., p. 596-603). Cf. MonNERIE 2012, sur la nécessité de prendre en considération les « actes verbaux » et les « actes nonverbaux » dans l'analyse d'une cérémonie donnée.

[14] « Le rite s'inscrit dans la vie sociale par le retour des circonstances appelant la répétition de son effectuation. Il se caractérise par des

\section{LE RITE DANS SES ESPACES}

La première section propose deux articles qui, mis en regard, présentent deux cas de figure tout à fait différents de sources disponibles pour aborder la question du rituel dans un espace et un contexte social donnés. Jonathan Engel expose les résultats préliminaires d'un travail de recherche. Il fait une proposition de méthode pour identifier le «potentiel symbolique » du matériel trouvé dans I'habitat rhénan à l'âge du Fer. Il s'agit là d'un préalable nécessaire à toute réflexion sur les rites domestiques, c'est-à-dire sur des rites exécutés dans un espace qui n'est pas entièrement voué à ces pratiques. À I'opposé, Laetitia Martzolff propose un panorama synthétique des sources, nombreuses, à la disposition du chercheur, sur la question des divers rituels dans le contexte des grands sanctuaires égyptiens [17]. Ces édifices sont conçus comme la maison du dieu et abritent une parcelle de la présence divine matérialisée sur terre par l'effigie honorée dans le temple. Si l'enceinte du sanctuaire abrite des activités de nature proprement artisanales et économiques, l'essentiel des activités est toutefois tourné vers l'entretien rituel de l'effigie divine. Les sources en ce domaine sont donc à la fois pléthoriques et de nature variée (archéologiques, iconographiques et textuelles). Le lecteur non égyptologue pourrait considérer que l'accès aux rites égyptiens [18] anciens dans un tel contexte est aisé. Toutefois, si le caractère « rituel » de ces sources n'est pas difficile à déterminer, leur traitement nécessite des précautions méthodologiques spécifiques d'un autre ordre: prise en considération de la chronologie respective des sources mises à contribution pour la restitution d'un rite donné dans le contexte d'une civilisation de 3000 ans d'histoire, approche critique des sources iconographiques dans un contexte où l'image, loin de chercher à reproduire plus ou moins fidèlement une situation rituelle, est d'abord une reconstruction idéologique, ou encore prise en compte du statut différencié des textes rituels connus, depuis les copies «sacralisées » en hiéroglyphes sur les parois des

procédures dont il implique la mise en œuvre afin d'imposer sa marque au contexte que son intervention même contribue à définir. » (SмITH 2010, p. 630)

[15] Voir Houseman 2001. Cf. Houseman 2012.

[16] SмITH 2010, p. 630.

[17] Pour une présentation générale des rites égyptiens et de leurs sources dans d'autres contextes (funéraire, domestique, et contexte de temples) et avec un autre type d'approche, voir STEVENS 2011 (qui présente une classification des rites égyptiens en : «Rite of Exchange and Communion », « Rites of Affliction », « Rites of Passage »).

[18] Pour une présentation générale de la ritualité dans les temples égyptiens, Coulon 2010. Voir encore EATON 2013. 
monuments jusqu'aux manuscrits de manuels rituels en écriture cursive, parvenus jusqu'à nous par divers biais [19].

\section{AUTOPSIE DE RITES : DU TERRAIN AUX TEXTES}

La deuxième partie regroupe deux présentations qui traitent du statut de l'animal dans les pratiques rituelles égyptiennes. Dans l'article « Harpocrate au chien et les cadavres de Qasr 'Allam. Perspectives sur le statut rituel des inhumations animales dans l'Égypte ancienne », Frédéric Adam, Frédéric Colin et Ivana Pranjic proposent une étude sur la présence des chiens dans la nécropole de Qasr 'Allam (Égypte, Bahariya) aux côtés de corps humains. Partant de l'analyse minutieuse des données de terrain (taphonomie et archéozoologie), l'article procède à une vaste mise en perspective avec d'autres sources, en particulier textuelles, afin de tenter de déterminer si I'archéologue est, sur son terrain, face à des sépultures d'animaux domestiques ou des restes d'un type de sacrifice peu attesté en Égypte ancienne. L'enquête intéressera autant les égyptologues que les historiens travaillant sur le statut des animaux dans les pratiques rituelles, par sa réflexion approfondie et sa méthodologie, mêlant données de terrains, mises en regard avec des sources textuelles et utilisation combinée, dans I'analyse, du point de vue de l'observateur moderne (approche étique) et des catégories égyptiennes qui sont loin de recouper nécessairement les nôtres (approche émique). L'article de Françoise Labrique («Le taurillon exclu du sacrifice ») permet de prolonger la réflexion sur le statut des animaux, «sujets » ou « objets » du rite selon les cas, à travers une analyse précise d'une inscription épigraphique de la Porte d'Évergète à Karnak. Ces deux contributions montrent que la question du statut des animaux dans les rituels est loin d'être évidente et qu'elle mérite un examen des sources attentif au moindre détail. Ce questionnement dépasse bien évidemment le cadre des contextes chronologiques et culturels abordés par ces deux contributions. À l'intérieur même de ce dossier, il est évoqué par Aurélie Roche à propos de pratiques prédynastiques et archaïques égyptiennes, par Jonathan Engel à propos de certains restes fauniques dans les habitats de l'âge du Fer européen examinés, et il rejoint les réflexions d'Anne Jacquemin sur la compréhension du sacrifice en Grèce ancienne.

[19] Voir les éclairantes remarques sur les sources égyptiennes, dans MyNOLOPOUlos \& RoEder (éd.) 2006, p. 18-19.

[20] Sur la notion de « trace » centrale dans la recherche historique de manière générale et en archéologie en particulier, voir PAILLER 2013.

\section{L'INDICE ICONOGRAPHIQUE EN CONTEXTE}

La troisième section est constituée dans ce dossier d'une contribution qui permet de rappeler la place essentielle de l'iconographie. Dans son texte « Et le roi tua I'hippopotame. Enquête sur les origines d'un rite égyptien », Aurélie Roche procède à une combinatoire des sources, mettant en relation motifs iconographiques prédynastiques (IVe millénaire) de la chasse à I'hippopotame et restes fauniques. Son objectif est de mettre en lumière les origines d'un rituel royal attesté par les sources épigraphiques archaïques et dans l'imagerie pharaonique, et d'en proposer une interprétation dans le cadre de l'idéologie royale. Son texte pose par ailleurs la question de la valeur historique de ces témoignages iconographiques. Pour compléter cette courte section dédiée aux sources iconographiques, le lecteur pourra mettre la contribution d'Aurélie Roche en regard avec certains développements que Laetitia Martzolff consacre à la décoration des temples égyptiens comme sources sur les rites. Ces deux textes questionnent, à leur manière, la valeur documentaire d'une image.

\section{AUX LIMITES DE LA TRACE MATÉRIELLE}

La quatrième section interroge les limites de la notion (au cœur de l'archéologie du rite) de trace [20] archéologique, dans sa définition, ainsi que dans sa capacité à rendre compte d'un rite. En prenant l'exemple des lettres aux morts égyptiennes, la contribution de Sylvie Donnat rappelle que l'écrit ne doit pas être, sans examen critique, rangé dans la catégorie des sources extérieures au rite. En tant que trace d'un geste graphique sur un support, l'écrit est aussi un vestige matériel (archéologique). Il peut donc être considéré comme une trace directe de rituel quand ce geste graphique est pris dans la procédure rituelle ou quand l'objet porteur d'une inscription est manipulé au cours d'une cérémonie. Ces considérations sur le statut de l'écrit dans le rite s'inscrivent dans une réflexion sur la relation entre l'écriture et le rite qui dépasse le cadre disciplinaire égyptologique [21], même si l'Égypte ancienne constitue un terrain propice à ce type d'enquête. Au sein du dossier, on pourra noter que ces questions rencontrent un écho dans la distinction opérée par Frédéric Colin entre les textes « produits du rituel » et les textes « discours sur le rituel ». L'introduction d'un autre volume

[21] Voir Koch-Piettre \& Batsch (éd.) 2010 ; Cartry, Durand \& KochPIETTRE (éd.) 2010, en particulier les contributions regroupées dans la troisième partie de l'ouvrage intitulée «Écrire ». Cf. encore BELAYCHE, MASSA \& CUSUMANo 2013 (avec un autre type d'approche). 
consacré à l'archéologie et au rituel en Égypte et dans le monde grec, dirigé par J. Mylonopoulos et $\mathrm{H}$. Roeder, établit pour sa part, au sein des textes rituels égyptiens, une distinction entre des textes prescriptifs (präskriptiven), descriptifs (deskriptiven), et enfin « opératoires » (operativen), c'est-à-dire directement impliqués dans la procédure rituelle, en tant que textes supports à une énonciation, mais aussi éventuellement comme textes écrits participant du rituel en tant qu'inscriptions [22]. Ces différentes approches soulignent I'intérêt de cette question épistémologique. Dans une tout autre perspective, la contribution de Philippe Quenet permet d'introduire une approche critique de la notion « d'indice matériel ». Le texte interroge l'apport, certes essentiel pour la reconstitution des gestes, mais généralement insuffisant pour l'interprétation, de la trace archéologique. Dans le cas de figure de la fosse de huit mètres de profondeur, creusée et remblayée avec du sable, des fondations du Temple ovale de Khafaje (Irak, III ${ }^{\mathrm{e}}$ millénaire av. J.-C.), le caractère démesuré de l'entreprise, qui ne paraît justifié par aucune contrainte technique, peut donc être considéré comme l'indice d'une pratique d'ordre symbolique. Mais s'ouvre ensuite, pour I'historien, le difficile travail de restitution de la portée

[22] Mynolopoulos \& Roeder 2006, p. 18. Une classification plus développée des différentes relations entre textes et rite est présentée et discutée dans DüCKER \& RoEDER (éd) 2004. Pour une définition de la catégorie des textes « opératoires », voir ibid., p. 15-18, et p. 27-36 pour l'application de ce type aux sources égyptiennes. sémantique (par définition «immatérielle ») de la pratique, en l'absence de référent textuel (descriptif, prescriptif ou de l'ordre du commentaire) évident. Partant de I'analyse précise des données du terrain, la contribution procède donc à une combinatoire des sources, convoquant parallèles architecturaux mieux documentés et textes pour tenter de comprendre la pratique.

\section{DE LA TRACE À L'INTERPRÉTATION}

Enfin, la contribution d'Anne Jacquemin « Le sacrifice dans le monde grec et ses interprétations » permet de clore ce dossier, en mettant cette fois le curseur non pas sur l'identification et le traitement des traces et indices du rite, mais sur le processus d'interprétation du chercheur. Sa contribution sur le sacrifice grec revient sur les différentes grilles utilisées pour la compréhension des sources textuelles et les interprétations parfois divergentes de ce rite central qu'elles ont pu générer. Soulignant l'intérêt porté actuellement aux « réalités matérielles » fournies par le terrain, qui permettent d'éclairer concrètement les données textuelles et iconographiques, son texte est un rappel de la nécessité, pour le chercheur, d'opérer d'incessants aller-retours entre l'examen minutieux de première main des sources et théories interprétatives qui permettent de renouveler les approches, une dialectique qui est au cœur du travail quotidien en sciences humaines.

Ambos Cl. \& Verderame L. (éd.) 2013, « Approaching Rituals in Ancient Cultures. Proceedings of the Conference, November 28-30, 2011, Roma = Questioni di rito: rituali come fonte di conoscenza delle religioni e delle concezioni del mondo nelle culture antiche », Rivista degli studi orientali nuovaserie 86.

Belayche N., Massa Fr. \& Cusumano N. (éd.), 2013, « Écrire dans les pratiques rituelles de la Méditerranée antique. Identités et autorités », Revue d'Histoire des Religionsfasc. 2.

Carastro M, Guenzi C. \& Ildefonse Fr., 2012, «Pratiquer le comparatisme : textes, terrains, artefacts », La Lettre de l'EHESS 57. En ligne. URL : http://lettre.ehess.fr/4763 (consultation juin 2014).

Coulon L., 2010, « Ancienne Égypte : les rites », dans G. Andreu-Lanoë (éd.), Inventaire de l'Égypte (coll. Inventaires-Universalis), Paris, p. 283-289.

Detienne M. \& Vernant P. (éd.), 1979, La Cuisine du sacrifice en pays grec, Paris.

DüCKER B. \& ROEDER H., 2004, Rituelle Texthandlungsklassen: interdisziplinäre Betrachtungen zum Verhältnis von Text und Ritual(Forum Ritualdynamik 8), Heidelberg (URL: http://www.ub.uni-heidelberg.de/archiv/4958; consultation juillet 2014) 
Eaton K., 2013, Ancient Egyptian Temple Ritual. Performance, Pattern and Practice, New York - London.

Houseman M., 2001, « Was ist ein Ritual? », dans J.H. Martin et al., Altäre. Kunstzum Niederknien, Ostfildern-Ruit, 2001, p. 48-51.

Houseman M., 2012, Le rouge est le noir. Essais sur le rituel, Toulouse.

Insoll Th. (éd.), 2011, The Oxford Handbook of the Archaeology of Ritual and Religion, Oxford - New York.

Koch-Piettre R. \& Batsch Chr.(éd.), 2010, « Écritures rituelles », Cahiers « Mondes anciens » [En ligne], 1| 2010, mis en ligne le 01 janvier 2010, consulté le 25 avril 2014. URL : http://mondesanciens.revues.org/128.

Kyriadis E., 2005, Ritual in the Bronze Age Aegean, London.

Lepetz S. \& VAn Andringa W. (éd.), 2008a, Archéologie du sacrifice animal en Gaule romaine. Rituels et pratiques alimentaires, Montagnac.

Lepetz S. \& Van Andringa W., 2008b, « Archéologie du rituel. Méthode appliquée à l'étude de la nécropole de Porta Nocera à Pompéi », dans J. Scheid (éd.) Pour une archéologie du rite. Nouvelles perspectives de l'archéologie funéraire (Collection de l'Ecole française de Rome 407), Rome, p. 105-126.

Levi-Strauss Cl., 1971, L'Homme nu, Paris.

Monnerie D., 2012, «Objets cérémoniels, chaînes opératoires et élaboration des relations sociales (Arama et Hoot Ma Whaap, Kanaky Nouvelle-Calédonie, Mélanésie) 》, Techniques \& Culture 58, p. 122-141.

Mylonopoulos J. \& Roeder H. (éd.) 2006, Archäologie und Ritual: auf der Suche nach der rituellen Handlung in den antiken Kulturen Ägyptens und Griechenlands, Wien.

Pailler J.-M., 2013, « Le goût de la trace. Pour une ichnosophie raisonnée », dans Fr. Janot \& G. Giuliato (éd.) Indices et traces. La mémoire des gestes. Actes du colloque international 16, 17 et 18 juin 2011, UFR d'Odontologie de I'Université de Lorraine, Nancy, p. 363-381.

Quantin Fr. (éd.), 2011, Archéologie des religions antiques. Contributions à l'étude des sanctuaires et de la piété en Méditerranée (Grèce, Italie, Sicile, Espagne), Pau.

Scherd J., 2000, «Pour une archéologie du rite », Annales. Histoires, sciences sociales, 55e année fasc. 3, p. 615-622.

Scheid J. (éd.), 2008, Pour une archéologie du rite. Nouvelles perspectives de l'archéologie funéraire (Collection de l'Ecole française de Rome 407), Rome.

Sмiтн P., 2010, «Rite », dans P. Bonte \& M. Izard (éd.) 2010, Dictionnaire de l'ethnologie et de l'anthropologie, $4^{\mathrm{e}}$ édition (1re éd. 1991), Paris, p. 630-633.

Stevens A., 2011, « Chapter 45. Egypt», dans Th. Insoll (éd.) The Oxford Handbook of the Archaeology of Ritual and Religion, Oxford - New York, p. 722-744.

Testart A., 2006, « Comment concevoir une collaboration entre anthropologie sociale et archéologie ? À quel prix ? Et pourquoi ? », Bulletin de la Société préhistorique française 13, p. 385-395.

Van Andringa W. \& Lepetz S., 2005, « Pompéi, Porta Nocera, Archéologie du rituel : Fouille de l'enclos 23 de la nécropole », MEFRA 117, p. 339-346.

Wilburn A.T., 2012, Materia magica. The Archaeology of Magic in Roman Egypt, Cyprus, and Spain, Ann Arbor. 